\section{Psychological Medicine}

cambridge.org/psm

\section{Original Article}

Cite this article: van der Zweerde $T$, van Straten A, Effting M, Kyle SD, Lancee J (2018). Does online insomnia treatment reduce depressive symptoms? A randomized controlled trial in individuals with both insomnia and depressive symptoms. Psychological Medicine 49, 501-509. https:// doi.org/10.1017/S0033291718001149

Received: 12 November 2017

Revised: 2 March 2018

Accepted: 11 April 2018

First published online: 11 May 2018

\section{Key words:}

CBT; depression; insomnia; online treatment; randomized controlled trial

Author for correspondence:

T. van der Zweerde,

E-mail: t.vander.zweerde@vu.nl (c) Cambridge University Press 2018. This is an Open Access article, distributed under the terms of the Creative Commons Attribution licence (http://creativecommons.org/licenses/ by/4.0/), which permits unrestricted re-use, distribution, and reproduction in any medium, provided the original work is properly cited.

\section{CAMBRIDGE} UNIVERSITY PRESS

\title{
Does online insomnia treatment reduce depressive symptoms? A randomized controlled trial in individuals with both insomnia and depressive symptoms
}

T. van der Zweerde ${ }^{1}$, A. van Straten ${ }^{1}$, M. Effting ${ }^{2}$, S. D. Kyle ${ }^{3}$ and J. Lancee ${ }^{2}$

${ }^{1}$ Department of Clinical Psychology \& EMGO Institute for Health and Care Research, VU University, Amsterdam, The Netherlands; ${ }^{2}$ Department of Clinical Psychology, University of Amsterdam, Amsterdam, The Netherlands and ${ }^{3}$ Sleep and Circadian Neuroscience Institute, University of Oxford, Oxford, UK

\begin{abstract}
Background. Insomnia is effectively treated with online Cognitive Behavioral Therapy for Insomnia (CBT-I). Previous research has suggested the effects might not be limited to sleep and insomnia severity, but also apply to depressive symptoms. Results, however, are mixed.

Methods. In this randomized controlled trial we investigated the effects of guided online CBT-I on depression and insomnia in people suffering from symptoms of both. Participants $(n=104)$ with clinical insomnia and at least subclinical depression levels were randomized to (1) guided online CBT-I and sleep diary monitoring (i-Sleep) or (2) control group (sleep diary monitoring only). The primary outcome was the severity of depressive symptoms (Patient Health Questionnaire-9 without sleep item; PHQ-WS). Secondary outcomes were insomnia severity, sleep diary parameters, fatigue, daytime consequences of insomnia, anxiety, and perseverative thinking.

Results. At post-test, participants in the i-Sleep condition reported significantly less depressive symptoms (PHQ-WS) compared with participants in the sleep-diary condition $(d=0.76)$. Large significant effects were also observed for insomnia severity $(d=2.36)$, most sleep diary parameters, daytime consequences of insomnia, anxiety, and perseverative thinking. Effects were maintained at 3 and 6 month follow-up. We did not find significant post-test effects on fatigue or total sleep time.

Conclusions. Findings indicate that guided online CBT-I is not only effective for insomnia complaints but also for depressive symptoms. The effects are large and comparable with those of depression therapy. Clinical trial registration number: NTR6049 (Netherlands Trial Register).
\end{abstract}

\section{Introduction}

Insomnia and depression are both prevalent, severe disorders. Insomnia is characterized by difficulty initiating or maintaining sleep for three nights per week for 3 months, causing distress and impaired daily functioning (American Psychiatric Association, 2013). Depression is diagnosed when someone experiences depressed mood and/or loss of interest in daily activities for 2 weeks and shows four or more other specific symptoms (e.g. inactivity, concentration problems) along with impaired functioning (APA, 2013). Insomnia and depression are highly disturbing to a person's life and present significant financial burdens to society due to increased care consumption and decreased work productivity (Léger et al., 2001; Daley et al., 2009; Watkins et al., 2009). Comorbidity is the rule rather than the exception; around $40 \%$ of people with insomnia suffer from clinical depression (Taylor et al., 2005, 2007) and around $80 \%$ of depressed individuals suffer from insomnia symptoms (Ohayon, 2002; Szuba et al., 2003; Franzen and Buysse, 2008).

Evidence is accumulating that insomnia plays a role in the development and maintenance of a depressive disorder. People with insomnia have a twofold chance of developing a depression (Baglioni et al., 2011; Li et al., 2018), insomnia perpetuates depressive disorders (Pigeon et al., 2008), and residual insomnia complaints predict relapse after depression (Carney et al., 2007). Suggested pathways through which insomnia affects depression include biological (e.g. sleep deprivation or fragmentation affecting the endocrine system), cognitive (e.g. effects of worrying and ruminating when lying awake), emotional (e.g. decreased emotional coping skills), and directly sleep-related pathways (e.g. use of sleep medication; Staner, 2010; Baglioni et al., 2014; Finan et al., 2015).

Despite the high comorbidity between insomnia and depression, it is still unclear which disorder should be treated (first) or whether combination treatments are more effective. 
What is clear is that an insomnia disorder is effectively treated with Cognitive Behavioral Therapy for Insomnia (CBT-I). Meta-analyses have demonstrated that the effects are rather small for total sleep time (TST; Hedges' $g=0.16$ ), but high for other sleep parameters such as sleep efficiency (SE; Hedges' $g=$ 0.71 ) and for insomnia severity (Hedges' $g=0.98$; Trauer et al., 2015; Wu et al., 2015; van Straten et al., 2017). Long-term effects of CBT-I surpass the effects of sleep medication (Smith et al., 2002; Riemann and Perlis, 2009). Because of growing demand for and shortage of trained therapists, as well as difficulties some patients encounter (e.g. travel time, disabilities) treatments are also offered online to enhance accessibility. Online CBT-I is administered effectively with effect sizes in the range of face-to-face treatment (Espie et al., 2014; van Straten et al., 2014; Zachariae et al., 2016; Ritterband et al., 2017), although direct comparisons between online and face-to-face CBT-I show mixed results (Blom et al., 2015b; Lancee et al., 2016). The effects of (online) CBT-I are not limited to sleep parameters and insomnia severity. Meta-analyses report moderate effects of insomnia treatment on comorbid depressive symptoms experienced by insomnia patients $(S M D=0.36$ in Ye et al., 2015; $d=0.34$ in Ballesio et al., 2017). However, most trials included in these meta-analyses did not target depressed populations specifically and in several studies patients with higher depression scores were excluded.

To our knowledge there have only been four studies that investigated CBT-I as a standalone treatment for people with both depressive symptoms and insomnia. Two of those studies used face-to-face treatment and both failed to demonstrate an effect of insomnia treatment on depressive symptoms compared with depression treatment-as-usual (Wagley et al., 2013) and compared with relaxation therapy (Norell-Clarke et al., 2015). The other two studies used online CBT-I as a standalone treatment for depressive symptoms and insomnia. Those studies showed more promising results. Blom and colleagues showed that online CBT-I was significantly more effective than online depression treatment on insomnia but as effective on depressive symptoms (Blom et al., $2015 a$ ). However, the sample size of this study was small and replication is needed before conclusions can be drawn. Christensen and colleagues (2016) demonstrated that online CBT-I effectively reduced depressive symptoms and insomnia symptoms in patients with complaints of both compared with an online placebo module. However, people with higher scores of depressive symptoms were excluded (Patient Health Questionnaire-9 >20; Spitzer et al., 1999).

We aimed to replicate the findings of Christensen and colleagues and expand them by not excluding people with higher levels of depressive symptoms, in a randomized controlled design comparing online CBT-I (i-Sleep) with a non-treated control group, monitoring their sleep daily. The online treatment included online feedback by a coach as this has been shown to potentiate efficacy (Lancee et al., 2013b). We hypothesized that i-Sleep would be more effective than the control group in reducing depression symptoms. Secondarily, we investigated effects on insomnia severity, sleep diary parameters, daytime consequences of insomnia, fatigue, anxiety, and perseverative thinking. We hypothesized that i-Sleep would be more effective compared with the control group. We hypothesized that the effects of i-Sleep are generalized on functioning and other psychiatric symptoms such as perseverative thinking and anxiety known to be related to insomnia (Ohayon and Roth, 2003; Ehring and Watkins, 2008; Harvey et al., 2017).

\section{Methods}

\section{Participants and recruitment}

Participants who expressed interest in participating in research through a website on insomnia (www.insomnie.nl) were recruited. Potential participants received an email invitation to a screening questionnaire. Recruitment was completed in October 2016. The last follow-up occurred in June 2017 (see Fig. 1).

Inclusion criteria (assessed in the online screening) were: (1) $\geqslant 18$ years, (2) fulfilling DSM-5 criteria for insomnia (APA, 2013): trouble falling or staying asleep, $\geqslant$ three nights a week, $\geqslant 30 \mathrm{~min}$, with significant consequences, sufficient opportunity to sleep and dissatisfaction with amount or quality of sleep; (3) depressive symptoms [PHQ-9 score >4; (Spitzer et al., 1999)]; and (4) access to the Internet and email. Exclusion criteria were (1) probable sleep apnea (Wilson et al., 2010); (2) previous CBT-I; (3) started psychotherapy < 6 months ago; (4) pregnancy/breastfeeding; (5) shift work; (6) being diagnosed with psychosis/schizophrenia; and (7) suicidal intentions [five items from the Mini International Neuropsychiatric Interview diagnostic interview (Sheehan et al., 1998)]. Other psychiatric and somatic comorbidities were allowed, as were the use of sleep medication, melatonin, and homeopathic aids.

\section{Sample size}

Based on the effect on depression (PHQ-9) of $d=0.69$ reported by Christensen et al. (2016), a desired power of 0.8 and an alpha level of 0.05 (two-sided) a total of 34 participants per condition was needed. Considering risk of high dropout from online interventions (24\% in Horsch et al., 2015) we aimed to include 52 participants per condition.

\section{Procedure}

After informed consent, participants completed the baseline questionnaire [demographics, Patient Health Questionnaire-9 (PHQ-9, Spitzer et al., 1999), Insomnia Severity Index (ISI, Bastien et al., 2001), consequences of insomnia during daytime (Espie et al., 2012); Fatigue Severity Scale (FSS, Krupp et al., 1989) and Hospital Anxiety and Depression Scale-Anxiety (HADS-A, Spinhoven et al., 1997)]. Due to technical issues, the Perseverative Thinking Questionnaire (PTQ, Ehring et al., 2012) scheduled at baseline was completed at week 1. Excluded participants were advised to contact their general practitioner. No applicants reported suicidal ideation. All eligible applicants were then asked to fill out a sleep diary. All participants that adhered to the diary (between 5 and 7 days) were included and subsequently randomized to (1) online CBT-I (i-Sleep) or (2) no treatment (sleep diary only). We used block randomization (blocks 2-4) with a 1:1 allocation sequence generated by an independent researcher also performing actual allocation. Blinding researchers or participants was not possible.

All participants were asked weekly to complete the PHQ-9 (Spitzer et al., 1999), the ISI (Bastien et al. 2001), and the questionnaire on daily consequences (Espie et al., 2012). In addition, we asked participants to complete a sleep diary every morning (Carney et al., 2012).

Post-assessments occurred 9 weeks after randomization, for testing the primary hypothesis. Intervention participants received a 3 and 6-month follow-up assessment. The control group received treatment after post-assessment (week 9). Figure 1 shows a flow 


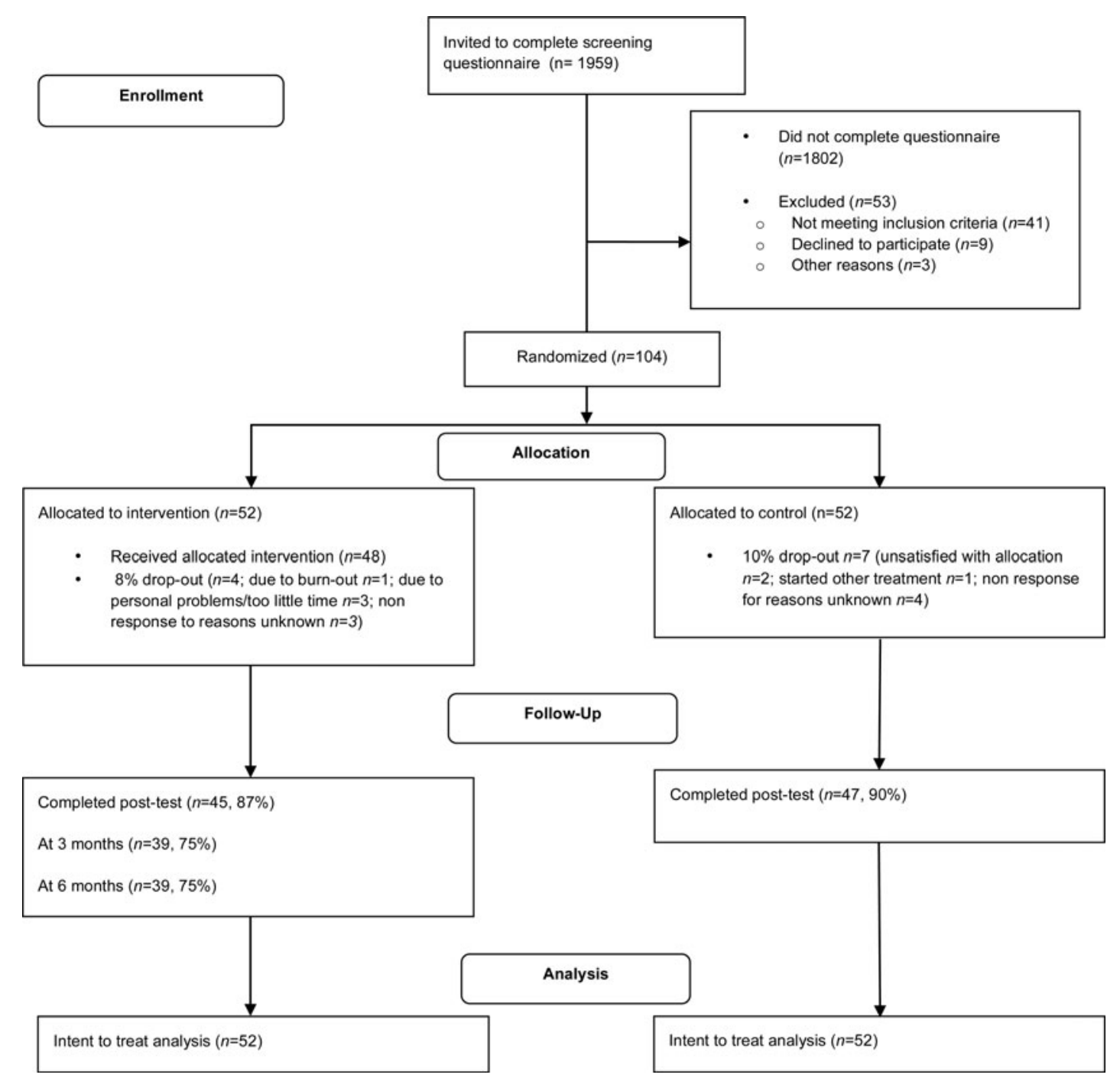

Fig. 1. CONSORT 2010 Flow Diagram.

chart of the study. The study was registered at the Netherlands Trial Register (NTR6049). The University of Amsterdam Ethics Review Board approved the protocol (2016-CP-7263).

\section{Intervention}

The online intervention i-Sleep consists of five sessions of CBT for insomnia (Morin and Espie, 2003; van der Zweerde et al., 2016). The 5 sessions focused on (1) sleep hygiene and lifestyle, (2) stimulus control and sleep restriction therapy [SRT; in which the time in bed (TIB) is restricted to the average time slept in the last week (with a 5-h minimum), increasing TIB when efficiency is $>85 \%$ and decreasing when it is $<80 \%$, with the aim of increasing the TIB spent asleep and decreasing TIB spent awake], (3) relaxation, (4) cognitive therapy tackling dysfunctional thoughts about sleep, (5) relapse prevention (van der Zweerde et al., 2016). Clinical psychology graduate students at the University of Amsterdam offered online guidance ( $\sim 40 \mathrm{~min}$ per participant per week) under weekly supervision by the first and last author. Online guidance entailed feedback on exercises, discussing SRT/bedtimes based on the diary and motivating participants to persevere in the treatment.

\section{Outcome measures}

The primary outcome measure was the Dutch version of a nineitem depression scale, the Patient Health Questionnaire-9 at week 9 (PHQ-9; range 0-27, Cronbach's $\alpha=0.94$; Spitzer et al., 1999). The PHQ-9 is scored on a five-point Likert score ranging from 0 (never) to 4 (almost daily). Next to the total score of the PHQ-9 we also report the total score without the sleep item, preventing measuring a decline in depression directly due to improved sleep (see also Christensen et al., 2016). We call this the Patient Health Questionnaire-9 without sleep item (PHQ-WS) score.

Secondary measures were included to assess insomnia symptoms, daytime functioning and other psychological symptoms associated with insomnia and depression. The Insomnia Severity Index (ISI; Bastien et al., 2001) is a seven item-scale scored on a five-point Likert scale. The total score ranges from 0 to 28 . The ISI has good psychometric properties (Cronbach's $\alpha=0.78$ ). The daytime consequences of insomnia were measured with six items (energy, mood, concentration, sleepiness, productivity, and relationships). Each item was scored on five-point Likert scale (Espie et al., 2012) and summed into one total score for daytime functioning. Fatigue was measured with the FSS (Cronbach's 
$\alpha=0.89$, nine statements scored on a seven-point Likert scale; Krupp et al., 1989). Anxiety was measured with the Anxiety section of the Hospital Anxiety and Depression Scale (HADS-A; Cronbach's $\alpha=0.84$, seven items on a four-point Likert scale, scores ranging 0-21; Spinhoven et al., 1997). Perseverative thinking was measured using the PTQ (Cronbach's $\alpha=0.94 ; 15$ items are rated on a four-point Likert scale, scores range 0-60; Ehring et al., 2012). For all questionnaires, higher scores indicated more severe complaints.

\section{Sleep diary}

The Carney consensus sleep diary (Carney et al., 2012) was slightly adjusted to fit our study purpose and reduce participant burden. Participants reported when they went to bed to sleep and when they got up, sleep onset latency (SOL), TIB awake after sleep onset (WASO), how long they slept in total and mood on a scale of 1-10 (higher scores indicating better mood). We calculated TST (TST: TIB-SOL-WASO), and then calculated the SE (SE: TST/TIB $\times 100$ ). Two items of the original Carney diary were left out: (1) the item distinguishing between the time patients went to bed and the time they switched off the light, and (2) the item distinguishing between final morning awakening and getting up.

\section{Adverse events}

At follow-up, participants were asked about adverse events over the course of participation. They indicated whether they had fallen, had an (traffic) accident or experienced other negative events that seemed related to fatigue and/or sleepiness (and if yes, what happened and any physical or psychological consequences).

\section{Statistical methods}

All randomized participants were included in the analyses following the intention-to-treat principle (Newell, 1992). Generalized mixed (multilevel) regression analysis was used to evaluate within-group effects (Time) and between-group effects (Time $\times$ Condition) of the intervention. Mixed negative binomial regression analyses with a log link were performed for skewed variables (PHQ-WS, ISI, PTQ, FSS, SE, SOL, WASO, TST), regular linear mixed regression with an identity link in all other cases. Two-level (participants and measurement points) repeatedmeasures designs were examined with outcomes as dependent variables (PHQ, PHQ-WS, ISI, daytime consequences, FSS, HADS-A, PTQ, sleep diary), Condition as between-subjects factor (intervention $v$. control) and Time as a within-subjects factor (pre- $v$. post-test). We used an unstructured covariance structure, which assumes data is missing at random, as the best model for the data and number of measurement points. Significance tests were performed to assess pre-treatment differences between groups. Baseline characteristics that significantly differed between groups and variables that predicted dropout were added as covariates. Analyses were repeated without covariates as a sensitivity analysis.

Within-group Cohen's $d$ effect sizes were based on multilevel estimated baseline and post-test means (i.e., pre-post) and pooled observed standard deviations at baseline. Between-group Cohen's $d$ effect sizes were calculated by dividing the difference in change scores (i.e., pre-post) by the pooled standard deviations at baseline (Morris, 2008). Cohen's $d$ was considered small (i.e. $<0.20$ ), moderate (around 0.50) or large $(\geqslant 0.80$; Cohen, 1988). Analyses were carried out using a $0.05 \alpha$-level (two-tailed) in SPSS v24.

\section{Results}

\section{Descriptives and non-response}

We included 104 patients (52 intervention and 52 control). The mean age of the sample was 45.99 years (s.D. $=12.32$ ) and $82 \%$ of the participants were female (Table 1). Insomnia duration was around 10 years $(M=9.79$, s.D. $=9.91)$. The majority of participants completed higher vocational or university education (60\%). At baseline, participants showed mild (PHQ-9>4) to severe depression (PHQ-9>19) with scores ranging from 5 to $22(\mathrm{M}=10.19$, S.D. $=3.90$; see online Supplementary Fig. $\mathrm{S} 1)$. There were no baseline differences between groups with the exception of perseverative thinking (see online Supplementary Table S4). Those in the i-Sleep condition reported more baseline perseverative thinking $(\mathrm{M}=32.35$, s.D. $=1.53)$ than participants in the control condition ( $M=28.69$, S.D. $=1.73 ; U=1568, p=0.03$ ). Age, depression scores (PHQ-9 and PHQ-WS), and TST proved to be associated with non-response at post-test in the treatment condition: younger patients, patients with higher depression scores and shorter TST were less likely to fill out the post-test. We included these variables as covariates in the regression models on all outcome measures. Non-response in the control condition was not related to any of the variables examined. At the followups, baseline PTQ was associated with nonresponse (nonresponders had more perseverative thinking) and was therefore included as a covariate in the analysis. Figure 1 shows a flowchart of the study.

\section{Treatment adherence, satisfaction, and adverse events}

Most of the 52 intervention participants $(n=41,85 \%)$ completed the full i-Sleep intervention. Four participants dropped out of the intervention (see flowchart, Fig. 1). The remaining participants completed $1(n=3), 2(n=1)$, or four sessions $(n=3)$.

On average patients were satisfied with the website $(\mathrm{M}=7.67$ on a scale $1-10$, S.D. $=0.98)$, with the feedback $(M=7.91$, S.D. $=$ $0.87)$, and the online module $(M=7.91$, S.D. $=0.82$, see online Supplementary Table S1). No adverse events related to the intervention or trial were reported.

Table 1. Demographics and pre-treatment characteristics

\begin{tabular}{lccc}
\hline & $\begin{array}{c}\text { i-Sleep } \\
(n=52) \\
\text { M (s.E. })\end{array}$ & $\begin{array}{c}\text { Sleep diary } \\
(n=52)\end{array}$ & \\
\hline Age $($ s.E. $)$ & Statistic \\
\hline & $44.64(1.82)$ & $46.29(2.09)$ & $\begin{array}{c}t(102)=-0.691, \\
p=0.49\end{array}$ \\
\hline $\begin{array}{l}\text { Insomnia duration } \\
\text { (years) }\end{array}$ & $9.92(1.56)$ & $9.10(1.38)$ & $U=1023.5, p=0.76$ \\
\hline $\begin{array}{l}\text { Female sex, } \\
n(\%)\end{array}$ & $43(80.8 \%)$ & $42(82.7 \%)$ & $\chi^{2}=0.064, p=0.80$ \\
\hline $\begin{array}{l}\text { Antidepressant use, } \\
n(\%)\end{array}$ & $8(15.4 \%)$ & $7(13.5 \%)$ & $\chi^{2}=0.078, p=0.78$ \\
\hline $\begin{array}{l}\text { Prescription sleep } \\
\text { med. use, } n \text { (\%) }\end{array}$ & $15(28.8 \%)$ & $15(28.8 \%)$ & $\chi^{2}=0.004, p=0.95$ \\
\hline
\end{tabular}




\section{Treatment effects on depression}

Multilevel regression analyses showed significant Time $\times$ Condition interaction effects for all three depression scores [PHQ- $9, F_{(1,81)}=$ 20.54, $p<0.001$ (Fig. 2); PHQ-WS, $F_{(1,82)}=14.38, p<0.001$, and the Mood item in the diary, $\left.F_{(1,83)}=13.34, p<0.001\right]$. This indicates participants in the i-Sleep condition experienced a greater decline in depressive symptoms at post-test than the control condition $\left(d_{\text {between }}=1.05\right.$ for the total PHQ, $d_{\text {between }}=0.76$ for the PHQ without the sleep item and $d_{\text {between }}=0.68$ for the Mood item). Details of the analysis are in Table 2 and online Supplementary Tables S2-S5.

\section{Treatment effects on insomnia and fatigue}

Significant Time $\times$ Condition interactions were also found for insomnia severity [ISI, $F_{(1,51)}=51.94, p<0.001$; Fig. 2] and for several sleep parameters from the diary [SE, $F_{(1,89)}=9.66, p<$ 0.001 , SOL, $F_{(1,70)}=20.12, p<0.001$, and WASO, $F_{(1,80)}=22.59$, $p<0.001]$. This indicates that participants in the i-Sleep condition experienced more sleep improvements than participants in the control condition: they report lower insomnia severity $\left(d_{\text {between }}=2.36\right)$, higher SE $\left(d_{\text {between }}=0.67\right)$, lower SOL $\left(d_{\text {between }}=\right.$ $0.69)$ and less time spent WASO $\left(d_{\text {between }}=0.60\right)$. No significant Time $\times$ Condition effect could be found for TST, $F_{(1,73)}=0.04$, $p=0.84$, nor for Fatigue Severity, $F_{(1105)}=1.43, p=0.24$.
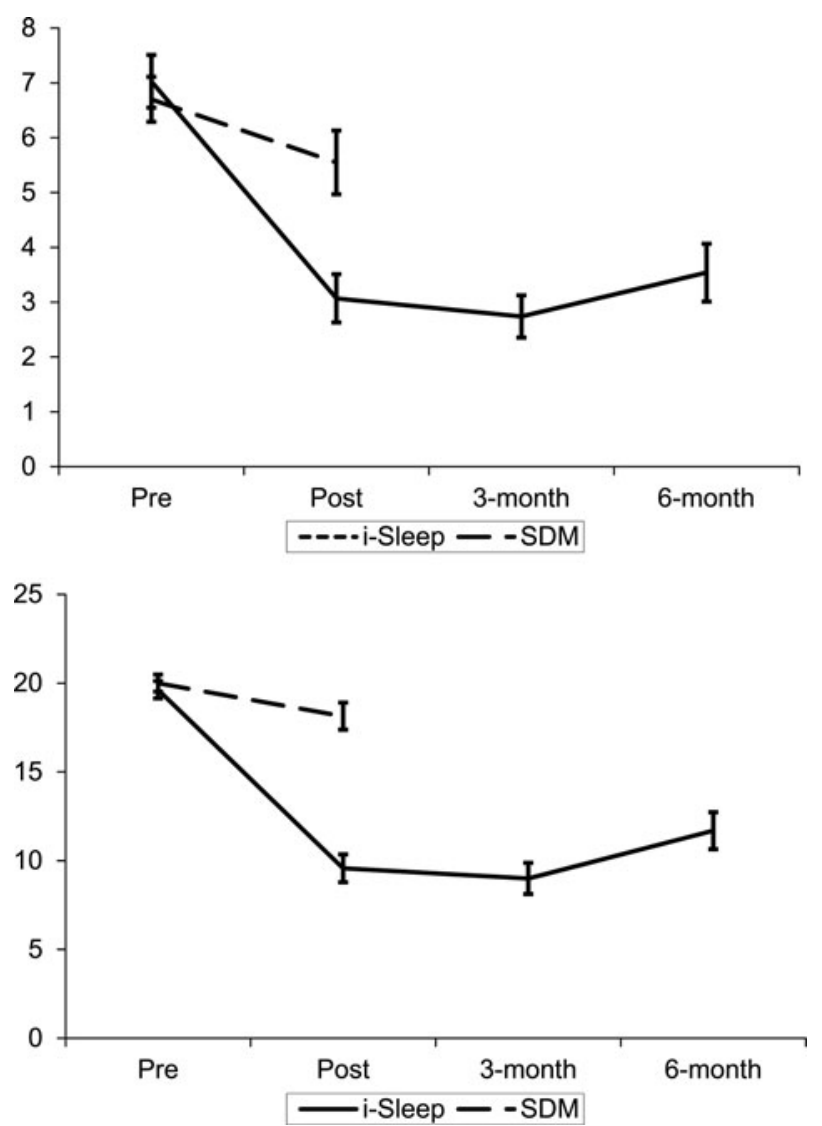

Fig. 2. Mean PHQ-WS and ISI in both conditions at pre- and post-assessment and FU for i-Sleep condition. Note: Error bars represent 95\% confidence intervals. PHQ-WS, Patient Health Questionnaire 9 minus the sleep item; ISI, Insomnia Severity Index; I-Sleep, online CBTI treatment; SDM, Sleep Diary Monitoring.
Treatment effects on daytime functioning, anxiety, and perseverative thinking

Significant Time $\times$ Condition interactions were found on anxiety [HADS-A, $\left.F_{(1,87)}=8.42, p<0.01\right]$ perseverative thinking [PTQ, $\left.F_{(1,91)}=9.48, p<0.01\right]$ and daytime functioning, $F_{(1,88)}=18.44$, $p<0.001$. We observed no differential effects for the separate aspects of the daytime functioning and hence only report the total score. Findings indicate that participants in the i-Sleep condition reported less anxiety $\left(d_{\text {between }}=0.63\right)$, less perseverative thinking $\left(d_{\text {between }}=0.62\right)$ and better daytime functioning $\left(d_{\text {between }}=1.12\right)$ than participants in the control group.

\section{Treatment effects at follow-up}

Non-significant time effects from post to follow-up assessments (using Greenhouse-Geisser correction where the sphericity assumption was violated) indicated that treatment effects at post assessment were largely maintained at follow-up assessment for depression [PHQ-9, $F_{(2,68)}=0.66, p=0.52$, PHQ-WS, $F_{(2,68)}=0.32, p=0.73$ ] and insomnia severity, $F_{(1.661,56,48)}=0.29, p=0.75$. Effects on daytime functioning was maintained, $F_{(2,68)}=0.073, p=0.93$ as well as on comorbid psychological symptoms [anxiety, $F_{(2,68)}=0.29, p=$ 0.77 , perseverative thinking, $\left.F_{(1.626,55.28)}=0.12, p=0.94\right]$ and several sleep parameters [TST, $F_{(1.275,15.30)}=2.09, p=0.15$, WASO, $F_{(2,20)}=$ $2.05, p=0.16$, SOL $F_{(2,22)}=2.49, p=0.11$ and SE, $F_{(2,18)}=2.60$, $p=0.10]$. There was no effect for fatigue at follow-up, $F_{(2,70)}=$ $0.93, p=0.4$, while mood improved further, $F_{(2,24)}=5.73, p=0.01$, $d=-0.23$.

\section{Clinically relevant improvements}

Participants in the i-Sleep condition showed significantly more clinically relevant improvements in both depression symptoms (clinical improvement defined as a drop of $50 \%$ or more resulting in <11; Spitzer et al., 1999; 64\% v. 30\%) and insomnia severity (clinical improvement defined as a change of $>8$; Morin et al., 2011; 64\% v. 9\%) than participants in the sleep diary monitoring control group. At 6 months follow-up, a considerable percentage of participants in the i-Sleep condition remained below the clinical cut-offs for depression (56\%) and insomnia severity (50\%) (see Table 3 for details).

\section{Discussion}

Our primary aim was to investigate effects of online CBT-I on depressive symptoms in a sample of people with insomnia and at least a subclinical level of depression. We hypothesized that the online intervention i-Sleep would be more effective than no treatment (sleep diary monitoring only). Our findings convincingly show online CBT-I, reduces symptoms of depression and insomnia in people experiencing both.

Overall, the large effect sizes that we observed on depressive symptoms (PHQ-9, $d_{\text {between }}=-1.05$; PHQ-WS, $d_{\text {between }}=-0.76$ ) contrast with two face-to-face studies published in the past, which did not observe an effect on depression (Wagley et al., 2013; Norell-Clarke et al., 2015). The effects we found are larger than those observed in two meta-analyses on effects of (online) CBT-I for depressive symptoms (SMD $=-0.36$; Ye et al., 2015a; $d=0.34$ for individual CBT-I, $d=0.13$ for self-help CBT-I; Ballesio et al., 2017) and larger than in the Christensen study which also specifically examined the effects of CBT-I on 
Table 2. (Mixed multilevel) regression-based pre, post, and FU estimated means and Cohen's $d$ effect sizes ${ }^{\star a}$

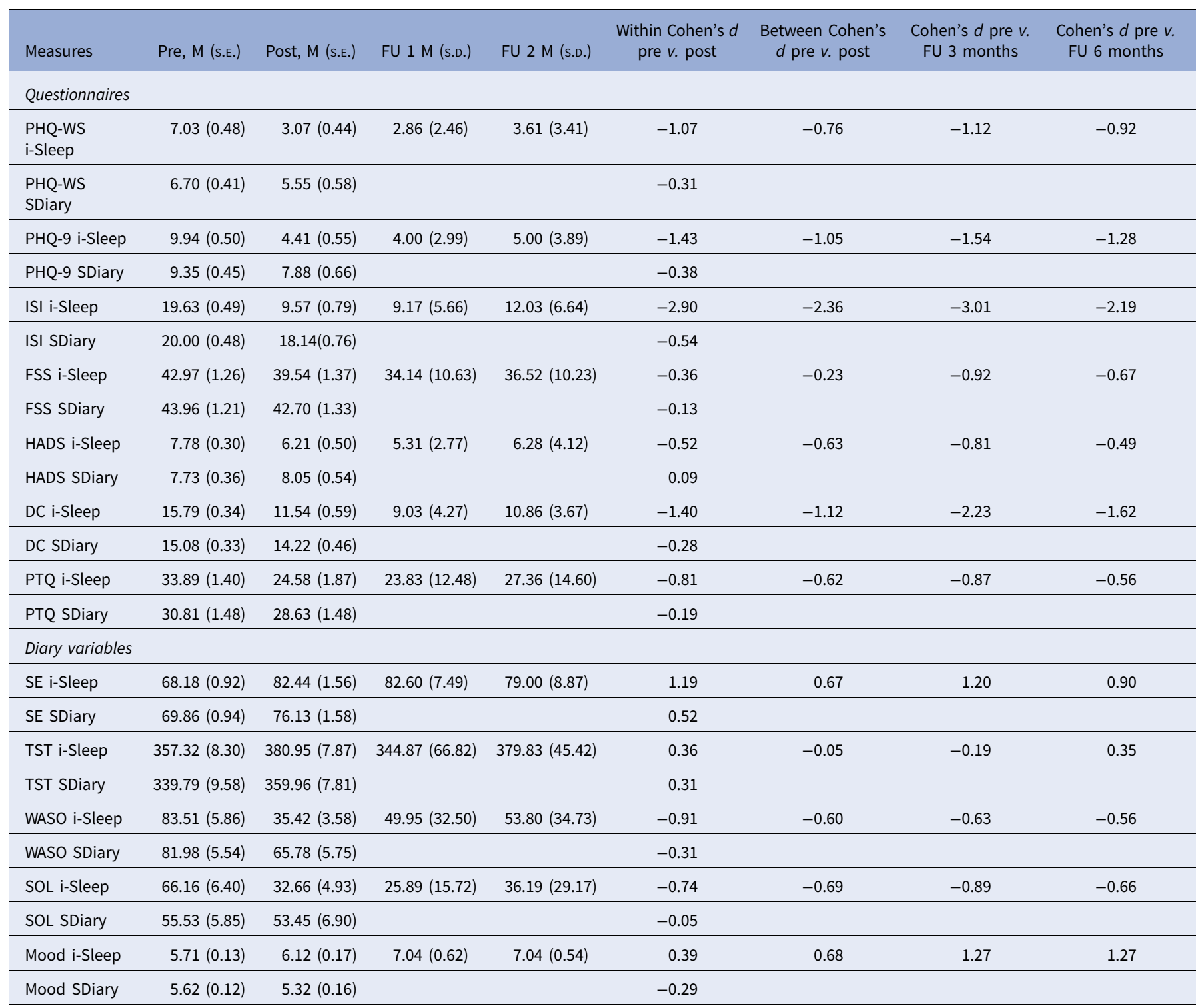

*A Cohen's $d$ is commonly considered to be either small (i.e. <0.20), moderate (around 0.50) or large (0.80 and over; Cohen, 1988).

DC, daytime consequences; FU, follow-up; FSS, Fatigue Severity Scale; HADS-A, Hospital Anxiety and Depression Scale-anxiety subscale; I, i-Sleep treatment condition; ISI, Insomnia Severity Index; PHQ-9, Patient Health Questionnaire-9; PHQ-WS, Patient Health Questionnaire without Sleep item; PTQ, Perseverative Thinking Questionnaire; SE, Sleep Efficiency; SDiary, sleep diary condition; SOL, Sleep Onset Latency; TST, Total Sleep Time; WASO, Wake After Sleep Onset.

${ }^{\mathrm{a} C}$ Cohen's $d$ using observed pooled standard deviation at baseline.

depression. There could be several reasons why our observed effect on depressive symptoms was better than in other studies. First, many studies in the meta-analyses did not specifically target depression. Second, we had higher baseline depression severity scores in our sample than in the Christensen trial, which provides more room for improvement. Also, we used personalized feedback while the Christensen trial, and some other studies included in the meta-analyses, used automated feedback. Personalized feedback has been shown to enhance treatment effects (Lancee et al., 2013a). Third and last, the Christensen trial used a more active control condition (Health Watch) than we did (monitoring only).

Our second aim was to explore effects of the treatment on insomnia. The observed effect on insomnia severity was comparable with a number of other online CBT-I trials but much larger $(d=-2.36)$ than what was observed in the trial by Christensen and colleagues $(d=-1.10,2016)$ and in the most recent meta-analysis of (online) CBT (Hedges' $g=1.03$ in Zachariae et al., 2016; Hedges' $g=0.98$ in van Straten et al., 2017). Future research is needed to identify sources of this heterogeneity of treatment effect sizes. The effects on SE were moderate, but in line with observations in a recent meta-analysis (van Straten et al., 2017). An increase in SE was also observed in the control condition (within Cohen's $d=0.52$, compared with $d=1.19$ in the i-Sleep condition). It appears that merely keeping a sleep diary can have a positive effect on SE, although this result is tentative since we did not include a third group who did not fill out a sleep diary. This means that we cannot rule out that this increase in SE might also be caused by natural recovery.

Interestingly, results did not show that participants were less fatigued or slept more (TST) compared with participants in the 
Table 3. Clinical improvement on depressive symptoms and insomnia severity

\begin{tabular}{|c|c|c|c|c|c|c|}
\hline & \multicolumn{2}{|c|}{ Baseline } & \multicolumn{2}{|c|}{ Post-test } & \multirow[b]{2}{*}{$\begin{array}{l}\text { Chi-square test of } \\
\text { conditions }\left(\chi^{2}\right)\end{array}$} & \multirow{2}{*}{$\begin{array}{l}6 \text { months follow-up } \\
\text { i-Sleep }(n=39)\end{array}$} \\
\hline & $\begin{array}{l}\text { i-Sleep } \\
(n=52)\end{array}$ & $\begin{array}{l}\text { Sleep diary } \\
(n=52)\end{array}$ & $\begin{array}{l}\text { i-Sleep } \\
(n=45)\end{array}$ & $\begin{array}{l}\text { Sleep diary } \\
(n=47)\end{array}$ & & \\
\hline PHQ-9 $>10^{\mathrm{a}}$ & $27(52 \%)$ & $21(40 \%)$ & $4(9 \%)$ & $13(28 \%)$ & $\chi^{2}(1)=5.38, p=0.02$ & $4(10 \%)$ \\
\hline PHQ $-9<5^{\mathrm{b}}$ & 0 & 0 & $28(62 \%)$ & $11(23 \%)$ & $\chi^{2}(1)=14.19, p<0.001$ & $22(56 \%)$ \\
\hline Clinically meaningful change PHQ-9c & & & $29(64 \%)$ & $14(30 \%)$ & $\chi^{2}(1)=11.09, p<0.001$ & $29(51 \%)$ \\
\hline$|S|>10^{d}$ & $52(100 \%)$ & $52(100 \%)$ & $17(38 \%)$ & $43(85 \%)$ & $\chi^{2}(1)=29.24, p<0.001$ & $19(50 \%)$ \\
\hline Clinically meaningful change $\mathrm{ISI}^{\mathrm{e}}$ & & & $29(64 \%)$ & $4(9 \%)$ & $\chi^{2}(1)=31.27, p<0.001$ & $29(56 \%)$ \\
\hline
\end{tabular}

${ }^{\mathrm{a}}$ Recommended cut-off point when using the PHQ-9 as a screener for depression (Kroenke and Spitzer, 2002).

${ }^{b}$ At least mild depressive symptoms, see inclusion criteria (Spitzer et al., 1999).

${ }^{c} \mathrm{PHQ}-9$ drop of $50 \%$ or more resulting in a score below 10 (Spitzer et al., 1999).

Note: at baseline, all participants had a PHQ-9 score of $\geqslant 5$ due to inclusion criteria.

${ }^{\mathrm{d}} \mathrm{A}$ clinical cut-off of 10 on the ISI is often used in insomnia research (Morin et al., 2011).

${ }^{\mathrm{e}} \mathrm{A}$ change of 8 points or more; considered to be a clinically meaningful change (Morin et al., 2011).

Note: At baseline, all participants had an ISI score of $>10$ due to the inclusion criteria.

control group. Perhaps, due to the SRT, participants were sleeping more efficiently and experiencing fewer symptoms (showing improvements on all symptom measures) at post-test, but were not recovered in such a way that they felt less tired. Possibly, the treatment causes participants to improve SE and therefore reduces insomnia complaints (i.e. decline on ISI, more satisfied with sleep, less trouble falling/staying asleep, etc.), while the SRT itself limited TST and actually caused fatigue. Fatigue is often an important reason for seeking treatment (Riedel and Lichstein, 2000) and is a commonly reported side effect of sleep restriction (Kyle et al., 2011). However, if the fatigue could be interpreted as a side effect of treatment here, then we would have expected to see postponed improvements in fatigue after treatment, in line with other research (e.g. Vitiello et al., 2014) we did not observe this after 6 months. Apparently insomnia severity and depression symptoms can largely improve despite fatigue remaining stable. Intuitively, it may seem that more and/ or better sleep should automatically lead to more restoration of depleted resources and therefore less fatigue. But research has shown the relationship between fatigue and sleep to be more complex (Fortier-Brochu et al., 2010). Future research may determine whether treatments targeting fatigue directly could be helpful in insomnia.

We found significant moderate to large improvements after online CBT-I treatment on our other outcomes intended to measure general (psychological) functioning: daytime functioning, anxiety, and perseverative thinking. It is interesting to note that although the treatment is not specifically aimed at these comorbid psychological symptoms, they do improve. The effect on daytime functioning (in energy, mood, concentration, sleepiness, productivity, and relationships) deserves specific attention because they are often overlooked in insomnia research (Kyle et al., 2010, 2013). Suffering daytime consequences is one of the main reasons for seeking treatment (Morin et al., 2006) and our findings suggest online CBT-I for people suffering from insomnia and depression has the potential to increase quality of life by improving daytime functioning. Future research will be necessary to confirm this (see e.g. protocol Espie et al., 2016).

CBT-I treatment might be regarded as a treatment not purely targeting insomnia. Some of the components are clearly targeting sleep, for example stimulus-control and SRT. However, parts of the sleep hygiene component (i.e., including promoting physical exercise and a structured routine in the morning and at night) show overlap with behavioral activation, a treatment component of CBT for depression. Additionally, targeting dysfunctional thoughts about sleep resembles cognitive therapy for depression and may encourage patients to use this strategy on non-sleep related depressive thought patterns as well. Likewise, relaxation is also used in depression treatment (Beck, 1979). The question remains whether depression symptoms abate because sleep improves, or whether specific components of the treatment work towards improving depression symptoms directly. Which specific CBT-I components improve depressive complaints remains to be investigated. Dismantling studies could be used to isolate treatment ingredients that confer benefit to sleep and depressive symptoms, which may help refine treatment of the common comorbid presentation. A proportion of the participants in our study that underwent treatment remained above clinical cut-offs for depression (38\%) and insomnia (38\%) at post-test. Future studies should investigate how, possibly, combining protocols may enhance treatment response. Additionally, mediation and network approach research is needed to see whether sleep improves before, after or at the same time as depression does.

Effects were maintained at follow-up. At 6 months follow-up, $22(56 \%)$ participants in the i-Sleep condition remained below the mild depression cut-off (PHQ-9 $<5$ ), and 19 participants $(50 \%)$ remained below the insomnia cut-off $(\leqslant 10)$ on the ISI. However, since we had a $25 \%$ non-response we cannot rule out that participants who did not complete the follow-up measurements were worse off than the ones that did (or neither that they were better off). Further research is needed to assess longerterm effects more reliably. Technical difficulties forced us to use a different program (Qualtrics, www.qualtrics.com) for the sleep diary at 6 months. This may have influenced the sleep diary adherence.

Some limitations of the present study need to be acknowledged. We actively recruited participants who were interested in insomnia treatment from the general population who were not clinically diagnosed for depression. The majority was female and highly educated. This is conceivably a very different population than patients that seek depression treatment through regular (mental) health care. However, this is also the case in other studies investigating the effect of online depression treatment on depressive symptoms that often use samples that show 
comparable baseline depression severity (e.g. Clarke et al., 2009; Cavanagh et al., 2011; Titov et al., 2015). The next step is to study whether online CBT-I treatment is also effective for patients with depressive symptoms in general (mental) health care settings, when patients turn to their general practitioner or psychologist for help. Also, in this study, we did not restrict time spent by clinical psychology students providing the feedback. Lancee et al. $(2013 a, 2013 b)$ showed it is possible to complete guidance for the entire treatment in $<40 \mathrm{~min}$. We expect trained professionals more experienced with patients will provide feedback in 1520 min per session, lowering the necessary investment.

In summary, our study shows that guided online CBT-I can be used to treat people suffering from both insomnia and depression symptoms. In our sample with mild to moderate depressive symptoms, effects on depressive symptoms (PHQ-9, $d=-1.05$; PHQ-WS, $d=-0.76$ ) were larger than those found in a metaanalysis of supported online CBT for self-reported depressive symptoms (Andersson and Cuijpers, 2009). Our findings provide further evidence that insomnia should not be treated as a mere symptom of a depressive episode but requires dedicated attention. Targeting insomnia may offer new potential in the treatment of depression. Further research is crucial to clarify the specific relations between insomnia and depression symptoms, in patients with a clinical diagnosis of both, preferably including a depression treatment condition and using both self-report and objective measures such as actigraphy, to optimize therapeutic effects and improve clinical outcomes.

Supplementary material. The supplementary material for this article can be found at https://doi.org/10.1017/S0033291718001149.

Acknowledgements. We thank our students Laura van Daal, Myrna Rood, Ilja Seifert, Anoushka Slieker, and Arved Stucke for their help with the treatments.

Author contributions. TZ, JL, ME, and AVS contributed to the study design. AS, JL, and TZ developed the intervention. TZ and JL carried out recruitment and data-collection. TZ performed analyses and drafted the manuscript. JL, $\mathrm{ME}$, and TZ supervised therapists. All contributed to and approved the manuscript.

Financial support. None.

\section{Conflict of interest. None.}

Ethical standards. The authors assert that all procedures contributing to this work comply with the ethical standards of the relevant national and institutional committees on human experimentation and with the Helsinki Declaration of 1975, as revised in 2008.

\section{References}

American Psychological Association (2013) Diagnostic and Statistical Manual of Mental Disorders, 5th Edn. Washington, DC: Author.

Andersson G and Cuijpers P (2009) Internet-Based and other computerized psychological treatments for adult depression: a meta-analysis. Cognitive Behaviour Therapy 38, 196-205.

Baglioni C et al. (2011) Insomnia as a predictor of depression: a meta-analytic evaluation of longitudinal epidemiological studies. Journal of Affective Disorders 135, 10-19.

Baglioni C et al. (2014) Sleep, depression and insomnia - a vicious circle? Current Psychiatry Reviews 10, 202-213.

Ballesio A et al. (2017) The effectiveness of behavioural and cognitive behavioural therapies for insomnia on depressive and fatigue symptoms: a systematic review and network meta-analysis. Sleep Medicine Reviews 37, $114-129$.
Bastien CH, Vallieres A and Morin CM (2001) Validation of the insomnia severity index as an outcome measure for insomnia research. Sleep Medicine 2, 297-307.

Beck AT (1979) Cognitive Therapy of Depression. New York: Guilford Press.

Blom K et al. (2015a) Internet treatment addressing either insomnia or depression, for patients with both diagnoses: a randomized trial. Sleep, 267-277.

Blom K et al. (2015b) Internet-vs. group-delivered cognitive behavior therapy for insomnia: a randomized controlled non-inferiority trial. Behaviour Research and Therapy 70, 47-55.

Carney CE et al. (2007) A comparison of rates of residual insomnia symptoms following pharmacotherapy or cognitive-behavioral therapy for major depressive disorder. The Journal of Clinical Psychiatry 68, 254-260.

Carney CE et al. (2012) The consensus sleep diary: standardizing prospective sleep self-monitoring. Sleep 35, 287-302.

Cavanagh K, Seccombe N and Lidbetter N (2011) The implementation of computerized cognitive behavioural therapies in a service user-led, third sector self-help clinic. Behavioural and Cognitive Psychotherapy 39, 427-442.

Christensen H et al. (2016) Effectiveness of an online insomnia program (SHUTi) for prevention of depressive episodes (the GoodNight study): a randomised controlled trial. The Lancet. Psychiatry 3, 333-341.

Clarke G et al. (2009) Randomized effectiveness trial of an internet, pure selfhelp, cognitive behavioral intervention for depressive symptoms in young adults. Cognitive Behaviour Therapy 38, 222-234.

Cohen J (1988) Statistical Power Analysis for the Behavioral Sciences, 2nd Edn. Chicago: Taylor \& Francis.

Daley M et al. (2009) Insomnia and its relationship to health-care utilization, work absenteeism, productivity and accidents. Sleep Medicine 10, 427-438.

Ehring T and Watkins ER (2008) Repetitive negative thinking as a transdiagnostic process. International Journal of Cognitive Therapy 1, 192-205.

Ehring T et al. (2012) Validation of the Dutch version of the perseverative thinking questionnaire (PTQ-NL). European Journal of Psychological Assessment 28, 102-108.

Espie CA et al. (2012) A randomized, placebo-controlled trial of online cognitive behavioral therapy for chronic insomnia disorder delivered via an automated media-rich web application. Sleep 35, 769-781.

Espie CA et al. (2014) Attribution, cognition and psychopathology in persistent insomnia disorder: outcome and mediation analysis from a randomized placebo-controlled trial of online cognitive behavioural therapy. Sleep Medicine 15, 913-917.

Espie CA et al. (2016) Digital cognitive behavioural therapy for insomnia versus sleep hygiene education: the impact of improved sleep on functional health, quality of life and psychological well-being: study protocol for a randomised controlled trial. Trials 17, 257.

Finan PH, Quartana PJ and Smith MT (2015) The effects of sleep continuity disruption on positive mood and sleep architecture in healthy adults. Sleep 38, 1735-1742.

Fortier-Brochu É et al. (2010) Relations between sleep, fatigue, and health-related quality of life in individuals with insomnia. Journal of Psychosomatic Research 69, 475-483.

Franzen PL and Buysse DJ (2008) Sleep disturbances and depression: risk relationships for subsequent depression and therapeutic implications. Dialogues in Clinical Neuroscience 10, 473-481.

Harvey AG et al. (2017) Mediators and treatment matching in behavior therapy, cognitive therapy and cognitive behavior therapy for chronic insomnia. Journal of Consulting and Clinical Psychology 85(10), 975-987. doi: $10.1037 /$ ccp0000244.

Horsch C et al. (2015) Adherence to technology-mediated insomnia treatment: a meta-analysis, interviews, and focus groups. Journal of Medical Internet Research 17, e214.

Krupp LB et al. (1989) The fatigue severity scale, application to patients with multiple sclerosis and systemic lupus erythematosus. Archives of Neurology 46, 1121-1123.

Kyle SD, Morgan K and Espie CA (2010) Insomnia and health-related quality of life. Sleep Medicine Reviews 14, 69-82.

Kyle SD et al. (2011) No pain, no gain: an exploratory within-subjects mixedmethods evaluation of the patient experience of sleep restriction therapy (SRT) for insomnia. Sleep Medicine 12, 735-747. 
Kyle SD et al. (2013) The Glasgow sleep impact index (GSII): a novel patientcentered measure for assessing sleep-related quality of life impairment in insomnia disorder. Sleep Medicine 14, 493-501.

Kroenke K and Spitzer RL (2002) The PHQ-9: a new depression diagnostic and severity measure. Psychiatric Annals 32, 509-515.

Lancee J et al. (2013a) Motivational support provided via email improves the effectiveness of internet-delivered self-help treatment for insomnia: a randomized trial. Behaviour Research and Therapy 51, 797-805.

Lancee J et al. (2013b) Motivational support provided via email improves the effectiveness of internet-delivered self-help treatment for insomnia: a randomized trial. Behaviour Research and Therapy 51, 797-805.

Lancee J et al. (2016) Guided online or face-to-face cognitive behavioral treatment for insomnia: a randomized wait-list controlled trial. Sleep 39, 183-191.

Léger D et al. (2001) SF-36: evaluation of quality of life in severe and mild insomniacs compared with good sleepers. Psychosomatic Medicine 63, 49-55.

Li MJ et al. (2018) Sleep and mood in older adults: coinciding changes in insomnia and depression symptoms. International Psychogeriatrics 30, 431-435.

Morin CM and Espie CA (2003) Insomnia: A Clinician's Guide to Assessment and Treatment, Vol. 1. New York: Springer.

Morin CM et al. (2006) Epidemiology of insomnia: prevalence, self-help treatments, consultations, and determinants of help-seeking behaviors. Sleep Medicine 7, 123-130.

Morin CM et al. (2011) The insomnia severity index: psychometric indicators to detect insomnia cases and evaluate treatment response. Sleep 34, 601608.

Morris SB (2008) Estimating effect sizes from pretest-posttest-control group designs. Organizational Research Methods 11, 364-386.

Newell DJ (1992) Intention-to-treat analysis: implications for quantitative and qualitative research. International Journal of Epidemiology 21, 837-841.

Norell-Clarke A et al. (2015) Group cognitive behavioural therapy for insomnia: effects on sleep and depressive symptomatology in a sample with comorbidity. Behaviour Research and Therapy 74, 80-93.

Ohayon MM (2002) Epidemiology of insomnia: what we know and what we still need to learn. Sleep Medicine Reviews 6, 97-111.

Ohayon MM and Roth T (2003) Place of chronic insomnia in the course of depressive and anxiety disorders. Journal of Psychiatric Research 37, 9-15.

Pigeon WR et al. (2008) Is insomnia a perpetuating factor for late-life depression in the IMPACT cohort? Sleep 31, 481-488.

Riedel BW and Lichstein KL (2000) Insomnia and daytime functioning. Sleep Medicine Reviews 4, 277-298.

Riemann D and Perlis ML (2009) The treatments of chronic insomnia: a review of benzodiazepine receptor agonists and psychological and behavioral therapies. Sleep Medicine Reviews 13, 205-214.

Ritterband LM et al. (2017) Effect of a web-based cognitive behavior therapy for insomnia intervention with 1-year follow-up: a randomized clinical trial. JAMA Psychiatry 74, 68-75.

Sheehan D et al. (1998) Diagnostic psychiatric interview for DSM-IV and ICD-10. Journal of Clinical Psychiatry 59, 22-33.
Smith MT et al. (2002) Comparative meta-analysis of pharmacotherapy and behavior therapy for persistent insomnia. American Journal of Psychiatry 159, 5-11.

Spinhoven PH et al. (1997) A validation study of the hospital anxiety and depression scale (HADS) in different groups of Dutch subjects. Psychological Medicine 27, 363-370.

Spitzer RL, Kroenke K, Williams JB and Patient Health Questionnaire Primary Care Study Group (1999) Validation and utility of a self-report version of PRIME-MD: the PHQ primary care study. Journal of the American Medical Association 282, 1737-1744.

Staner L (2010) Comorbidity of insomnia and depression. Sleep Medicine Reviews 14, 35-46.

Szuba MP, Kloss JD and Dinges DF (2003) Insomnia: Principles and Management. Cambridge: Cambridge University Press.

Taylor DJ et al. (2005) Epidemiology of insomnia, depression, and anxiety. Sleep 28, 1457-1464.

Taylor DJ et al. (2007) A pilot study of cognitive-behavioral therapy of insomnia in people with mild depression. Behavioral Therapy 38, 49-57.

Titov $\mathbf{N}$ et al. (2015) Mindspot clinic: an accessible, efficient, and effective online treatment service for anxiety and depression. Psychiatric Services 66, 1043-1050.

Trauer JM et al. (2015) Cognitive behavioral therapy for chronic insomnia: a systematic review and meta-analysis. Annals Internal Medicine 163, 191204.

van der Zweerde T et al. (2016) Cost-effectiveness of i-sleep, a guided online CBT intervention, for patients with insomnia in general practice: protocol of a pragmatic randomized controlled trial. BMC Psychiatry 16, 85.

Van Straten A et al. (2014) Guided internet-delivered cognitive behavioural treatment for insomnia: a randomized trial. Psychological Medicine 44, 1521-1532.

van Straten A et al. (2017) Cognitive and behavioral therapies in the treatment of insomnia: a meta-analysis. Sleep Medicine Reviews 38, 3-16.

Vitiello MV et al. (2014) Short-term improvement in insomnia symptoms predicts long-term improvements in sleep, pain, and fatigue in older adults with comorbid osteoarthritis and insomnia. Pain 155, 1547-1554.

Wagley J et al. (2013) Effectiveness of abbreviated CBT for insomnia in psychiatric outpatients: sleep and depression outcomes. Journal of Clinical Psychology 69, 1043-1055.

Watkins KE et al. (2009) The health value and cost of care for major depression. Value in Health 12, 65-72.

Wilson SJ et al. (2010) British association for psychopharmacology consensus statement on evidence-based treatment of insomnia, parasomnias and circadian rhythm disorders. Journal of Psychopharmacology 24, 1577-1601.

Wu JQ et al. (2015) Cognitive behavioral therapy for insomnia comorbid with psychiatric and medical conditions: a meta-analysis. JAMA Internal Medicine 175, 1461-1472.

Ye YY et al. (2015) Internet-based cognitive behavioral therapy for insomnia (ICBT-i) improves comorbid anxiety and depression: a meta-analysis of randomized controlled trials. PLoS ONE 10, e0142258.

Zachariae R et al. (2016) Efficacy of internet-delivered cognitive-behavioral therapy for insomnia: a systematic review and meta-analysis of randomized controlled trials. Sleep Medicine Reviews 30, 1-10. 